\author{
V. LE NIR, M. HELARD, R. LE GOUABLE
}

\title{
EFFICIENT DIVERSITY TECHNIQUES USING LINEAR PRECODING AND STBC FOR MULTI-CARRIER SYSTEMS
}

\section{INTRODUCTION}

Since the work of Foschini [1], there has been a huge interest concerning Multiple Input Multiple Output (MIMO) systems in order to exploit the capacity varying linearly with the minimum of transmit $\mathrm{N}_{t}$ and receive antennas $\mathrm{N}_{\mathrm{r}}$ and then to exploit the diversity of these systems using Orthogonal Space Time Block Codes (OSTBC) as discovered by Alamouti [2] for $\mathrm{N}_{t}=2$ and then generalized by Tarokh [3] for $2 \leq \mathrm{N}_{\mathrm{t}} \leq 4$. Quasi-Orthogonal (QO) STBC were then described in [4][5]. New codes are given in [6] for $\mathrm{N}_{\mathrm{t}}=5$ or 6 . In parallel, linear precoding was demonstrated to be very efficient in SISO transmission in order to exploit temporal diversity using Maximum Likelihood (ML) detector [7]. Using same type of detectors, linear precoders were adapated to multi-antenna transmissions. The linear precoders used as space-time codes were carried out in [8][9]. The concatenation of linear precoders with QOSTBC was carried out in [10]. In this paper, we combine a particular linear precoder with OSTBC in a specific way allowing a simple linear decoding for various cases of MIMO systems [11]. Several linear precoding matrices based on either Hadamard matrix or Fourier Transform construction are compared. In the second part, we present this linear precoding that has the effect of increasing the overall diversity of the system by scattering the information in the time and/or frequency domains for multi-carrier modulations. We apply our precoder to OFDM and MultiCarrier Code Division Multiplex Access (MC-CDMA) systems, exploiting spatial, temporal and frequency diversities.

\section{OSTBC REPRESENTATION}

The different channel coefficients are modelled as independent flat fading channels that are quite realistic for OFDM-like modulations. We consider uncorrelated channels from each transmit antenna $t$ to each receive antenna $r h_{t r}=\rho_{t r} e^{i \theta_{t r}}$. Assuming one receive antenna, the Alamouti code can be represented as follows:

$$
G_{2}=\left[\begin{array}{cc}
s_{1} & -s_{2}^{*} \\
s_{2} & s_{1}^{*}
\end{array}\right]
$$

Assuming fading coefficients constant over two consecutive symbol transmissions, the received signal over two consecutive symbols periods are:

$$
\left[\begin{array}{c}
r_{1} \\
-r_{2}^{*}
\end{array}\right]=\left[\begin{array}{cc}
h_{1} & h_{2} \\
-h_{2}^{*} & h_{1}^{*}
\end{array}\right] \cdot\left[\begin{array}{l}
s_{1} \\
s_{2}
\end{array}\right]+\left[\begin{array}{c}
n_{1} \\
-n_{2}^{*}
\end{array}\right]
$$

1

T.H.E. Editor(s) (ed.), Book title, $1-6$.

(C) yyyy Kluwer Academic Publishers. Printed in the Netherlands. 
where $n_{1}$ and $n_{2}$ are independent complex variables with zero mean and one-sided power spectral density $N_{0}$, representing Additive White Gaussian Noise (AWGN). For this study, perfect channel estimation is assumed. Applying the transpose conjugate of the channel matrix to the equivalent received vector, we obtain:

$$
\left[\begin{array}{l}
\hat{s}_{1} \\
\hat{s}_{2}
\end{array}\right]=\lambda \cdot I_{2} \cdot\left[\begin{array}{l}
s_{1} \\
s_{2}
\end{array}\right]+\left[\begin{array}{l}
h_{1}^{*} n_{1}+h_{2} n_{2}^{*} \\
h_{2}^{*} n_{1}-h_{1} n_{2}^{*}
\end{array}\right]
$$

with $\lambda=\left|h_{1}\right|^{2}+\left|h_{2}\right|^{2}$. This receiving process corresponds to a Maximum Ratio Combining (MRC) equalizer. However, an equalization process can be carried out according to the Zero Forcing or Minimum Mean Square Error criteria. This matrix representation can be extended for other OSTBC schemes [3][6] where we obtain:

$$
\lambda=\sum_{j=1}^{N_{t}}\left|h_{j}\right|^{2} \quad \text { or } \quad \lambda=\sum_{j=1}^{N_{t}}\left|h_{j}\right|^{2} /\left(\sum_{j=1}^{N_{t}}\left|h_{j}\right|^{2}+1 / \gamma\right)
$$

in the case of a MRC or a MMSE equalizer respectively, where $\gamma$ is the Signal to Noise Ratio at the receive antenna. We use different equalizers because they lead to different performance when using linear precoding.

\section{LINEAR PRECODING}

This linear precoding given in [11] is briefly presented. According to the theorem of diagonal decomposition, let $A_{L}$ be a Hermitian $L x L$ matrix with eigenvalues $\lambda_{1} \ldots \lambda_{\mathrm{L}}$. Then $A_{L}$ can be expressed as:

$$
A_{L}=\Theta_{L} \Lambda_{L} \Theta_{L}^{H}
$$

where $\Lambda_{L}=\operatorname{diag}\left(\lambda_{1}, \ldots, \lambda_{L}\right)$ and $\Theta_{L}$ is an unitary matrix so that $\Theta_{L}^{-1}=\Theta_{L}^{H}$, where (.) ${ }^{\mathrm{H}}$ stands for transpose conjugate. We propose to use the following linear precoding based on the Hadamard construction matrix such as:

$$
\Theta_{L}=\sqrt{\frac{2}{L}}\left[\begin{array}{cc}
\Theta_{L / 2} & \Theta_{L / 2} \\
\Theta_{L / 2} & -\Theta_{L / 2}
\end{array}\right]
$$

with $\mathrm{L}=2^{\mathrm{n}}, \mathrm{n} \in \mathbf{N}^{*}, \mathrm{n} \geq 2$ and:

$$
\Theta_{2}=\left[\begin{array}{cc}
e^{j \theta_{1}} \cdot \cos \eta & e^{j \theta_{2}} \cdot \sin \eta \\
-e^{j \theta_{2}} \cdot \sin \eta & e^{-j \theta_{1}} \cdot \cos \eta
\end{array}\right]
$$

belonging to the Special Unitary group $\mathrm{SU}(2)$, therefore $\operatorname{det}\left(\Theta_{2}\right)=1$. This leads to the following expression:

$$
A_{L}=\frac{2}{L}\left[\begin{array}{ll}
A_{L / 2}^{1}+A_{L / 2}^{2} & A_{L / 2}^{1}-A_{L / 2}^{2} \\
A_{L / 2}^{1}-A_{L / 2}^{2} & A_{L / 2}^{1}+A_{L / 2}^{2}
\end{array}\right]
$$


with $A_{L / 2}^{1}=\Theta_{L / 2} \Lambda_{L / 2}^{1} \Theta_{L / 2}^{H}$ and $A_{L / 2}^{2}=\Theta_{L / 2} \Lambda_{L / 2}^{2} \Theta_{L / 2}^{H}$, and

$$
\Lambda_{L}=\operatorname{diag}\left(\Lambda_{L / 2}^{1}, \Lambda_{L / 2}^{2}\right)
$$

where $\Lambda_{L / 2}^{1}=\operatorname{diag}\left(\lambda_{1}, \ldots, \lambda_{L / 2}\right)$ and $\Lambda_{L / 2}^{2}=\operatorname{diag}\left(\lambda_{L / 2+1}, \ldots, \lambda_{L}\right)$. For $L=2$, we obtain the following Hermitian matrix:

$$
A_{2}=\left[\begin{array}{cc}
\cos ^{2} \eta \cdot \lambda_{1}+\sin ^{2} \eta \cdot \lambda_{2} & -\cos \eta \cdot \sin \eta \cdot e^{j\left(\theta_{1}+\theta_{2}\right)} \cdot\left(\lambda_{1}-\lambda_{2}\right) \\
-\cos \eta \cdot \sin \eta \cdot e^{-j\left(\theta_{1}+\theta_{2}\right)} \cdot\left(\lambda_{1}-\lambda_{2}\right) & \sin ^{2} \eta \cdot \lambda_{1}+\cos ^{2} \eta \cdot \lambda_{2}
\end{array}\right]
$$

Therefore, one can see that for $A_{L}$ the diagonal elements are equal to:

$$
A_{i i}=\frac{2}{L} \sum_{k=0}^{L / 2-1}\left(\cos ^{2} \eta \cdot \lambda_{(2 k+1)}+\sin ^{2} \eta \cdot \lambda_{(2 k+2)}\right) \quad \forall i \in[1 \ldots L]
$$

and that some non-diagonal elements are similar to:

$$
A_{i j}=-\frac{2}{L} \cos \eta \cdot \sin \eta \cdot e^{-j\left(\theta_{1}+\theta_{2}\right)} \sum_{k=0}^{L / 2-1}\left(\lambda_{(2 k+1)}-\lambda_{(2 k+2)}\right)
$$

Owing to (8) form, the other terms of interference are also sum of difference between eigenvalues. By simulation, the optimal results were found for pure real or pure imaginay interference. For $L=2, \eta=\pi / 4, \theta_{2}=\theta_{1}-\pi / 2, \theta_{1}=5 \pi / 4$, we get:

$$
A_{2}=\frac{1}{2}\left[\begin{array}{ll}
\lambda_{1}+\lambda_{2} & \lambda_{2}-\lambda_{1} \\
\lambda_{2}-\lambda_{1} & \lambda_{1}+\lambda_{2}
\end{array}\right]
$$

One may use Fourier matrices instead of these matrices based on Hadamard construction, but we will see in the following section that they lead to worse performance for low values of $\mathrm{L}$ when the linear precoding is combined with STBC.

\section{LINEAR PRECODING WITH STBC}

In this paper, we combine the OSTBC with linear precoding by concatenation according to the equation (5) where $\Lambda_{L}$ represents the OSTBC coding and decoding without noise, and $\Theta_{\mathrm{L}}$ stands for the linear precoding. It is possible to use more transmit antennas by applying the OSTBC using subgroups of the available transmit antennas [11]. For instance, if we use the Alamouti code with four antennas, the first subgroup including antenna 1 and 2 will code the symbols according to Alamouti while antenna 3 and 4 are switched off. The total transmit power should remain $P$, therefore antenna 1 and 2 will transmit symbol at a power of $P / 2$. Then, antennas 3 and 4 will transmit the coded symbols according to Alamouti while antennas 1 and 2 are switched off. All OSTBC can be applied to this scheme as those described in [2][3][6] thus many scenarios can be drawn from this example depending on antenna configurations. As presented in Figure 1, at transmission side, input bits are first mapped into symbol vector $X=\left[\begin{array}{lll}x_{1} & \ldots & x_{L}\end{array}\right]$ where $L$ is the number of transmitted 
symbols. Linear precoding is then performed by applying the $\Theta_{L}$ matrix to the $X$ vector. The next step consists in applying an OSTBC to the symbol-rotated vector.

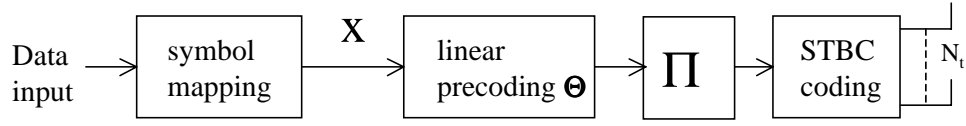

Fig.1 Combination of Linear Precoding with OSTBC: Transmitter scheme

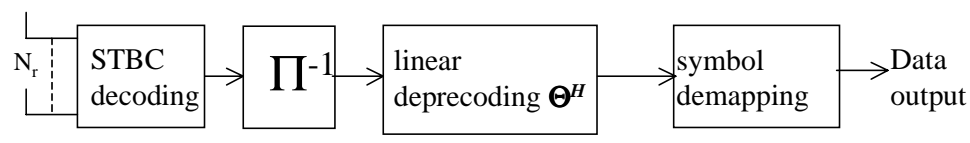

Fig.2 Combination of Linear Precoding with OSTBC: Receiver scheme

The receiver part is described by Figure 2. Without interleaving, the channel representation of the OSTBC codes leads to the equivalent channel coding and decoding matrix with the same diagonal elements. Owing to the Alamouti scheme (1) and the linear precoding described in (6) and (7) with optimal values given in Section 3, for $L=4$ without interleaving we get the following Hermitian matrix:

$$
\begin{gathered}
A_{4}=\Theta_{4} \Lambda_{4} \Theta_{4}^{H} \\
\text { with } \Lambda_{4}=\operatorname{diag}\left(\lambda_{1}, \lambda_{1}, \lambda_{2}, \lambda_{2}\right) \text { and } \lambda_{i}=\sum_{j=1}^{2}\left|h_{j}^{i}\right|^{2} \quad i \in[1 \ldots 2]
\end{gathered}
$$

where $i$ and $j$ are index used to distinguish different channels related by the OSTBC decoding.

The resulting matrix is then:

$$
A_{4}=\frac{1}{2}\left[\begin{array}{cccc}
\lambda_{1}+\lambda_{2} & 0 & \lambda_{1}-\lambda_{2} & 0 \\
0 & \lambda_{1}+\lambda_{2} & 0 & \lambda_{1}-\lambda_{2} \\
\lambda_{1}-\lambda_{2} & 0 & \lambda_{1}+\lambda_{2} & 0 \\
0 & \lambda_{1}-\lambda_{2} & 0 & \lambda_{1}+\lambda_{2}
\end{array}\right]
$$

When applying an interleaving process, the $\lambda$ 's within each block are affected by different channels:

$$
\lambda_{i}=\sum_{j=1}^{N_{t}}\left|h_{j}^{i}\right|^{2} \quad i \in[1 \ldots L]
$$

With interleaving, the $\Lambda_{4}$ matrix and the resulting in formula (14) becomes:

$$
\Lambda_{4}=\operatorname{diag}\left(\lambda_{1}, \lambda_{2}, \lambda_{3}, \lambda_{4}\right) \quad \text { with } \quad \lambda_{i}=\sum_{j=1}^{2}\left|h_{j}^{i}\right|^{2} \quad i \in[1 \ldots 4]
$$

Thus, the resulting matrix $A_{4}$ becomes: 
$A_{4}=\frac{1}{4}\left[\begin{array}{cccc}\lambda_{1}+\lambda_{2}+\lambda_{3}+\lambda_{4} & \lambda_{1}-\lambda_{2}+\lambda_{3}-\lambda_{4} & \lambda_{1}+\lambda_{2}-\lambda_{3}-\lambda_{4} & \lambda_{1}-\lambda_{2}-\lambda_{3}+\lambda_{4} \\ -\lambda_{1}+\lambda_{2}-\lambda_{3}+\lambda_{4} & \lambda_{1}+\lambda_{2}+\lambda_{3}+\lambda_{4} & -\lambda_{1}+\lambda_{2}+\lambda_{3}-\lambda_{4} & \lambda_{1}+\lambda_{2}-\lambda_{3}-\lambda_{4} \\ \lambda_{1}+\lambda_{2}-\lambda_{3}-\lambda_{4} & \lambda_{1}-\lambda_{2}-\lambda_{3}+\lambda_{4} & \lambda_{1}+\lambda_{2}+\lambda_{3}+\lambda_{4} & \lambda_{1}-\lambda_{2}+\lambda_{3}-\lambda_{4} \\ -\lambda_{1}+\lambda_{2}+\lambda_{3}-\lambda_{4} & \lambda_{1}+\lambda_{2}-\lambda_{3}-\lambda_{4} & -\lambda_{1}+\lambda_{2}-\lambda_{3}+\lambda_{4} & \lambda_{1}+\lambda_{2}+\lambda_{3}+\lambda_{4}\end{array}\right]$

One can notice that, with or without interleaver, at the receiver part a linear decoding can be performed by simply applying the transpose conjugate of the linear precoder. Therefore, we obtain a matrix of the form described in (14). The interleaving has the effect of mixing eigenvalues between different blocks, thus the components of the resulting matrix are different from each others. After OSTBC decoding and linear deprecoding, we can merely detect the signals. When $\mathrm{L}$ increases, OSTBC is performed on more subgroups and the resulting matrix corresponds to (5). With OSTBC, the diagonal elements follow a chi square law with $2 N_{t}$ degrees of freedom. When using linear precoding, the diagonal elements reach a chi square law with $N_{t} L$ degrees of freedom. With interleaving, the elements of the diagonal matrix reach a chi square law with $2 N_{t} L$ degrees of freedom, providing more diversity.

When using a Fourier matrix of size $L=4$ instead of the complex Hadamard matrix based on $\mathrm{SU}(2)$, we obtain a circulant matrix of the form:

$$
A_{4}=\frac{1}{4}\left[\begin{array}{llll}
a & b & c & d \\
d & a & b & c \\
c & d & a & b \\
b & c & d & a
\end{array}\right]
$$

with $a=\lambda_{1}+\lambda_{2}+\lambda_{3}+\lambda_{4}, b=\lambda_{1}-\lambda_{3}-j\left(\lambda_{2}-\lambda_{4}\right), c=\lambda_{1}-\lambda_{2}+\lambda_{3}-\lambda_{4}$ and $d=\lambda_{1}-\lambda_{3}+j\left(\lambda_{2}-\lambda_{4}\right)$. Hence, the interference terms are different from those obtained with the Hadamard construction. As L increases the interference terms will tend slower towards the gaussian law than the interference terms of the Hadamard construction presented before. Indeed, the interference terms of the Fourier construction follow a chi-square law with twice as less degrees of freedom as the Hadamard one per dimension.

\section{LINEAR PRECODING WITH STBC AND MULTI-CARRIER SYSTEMS}

In the precedent part, linear precoding is done in the time domain, but it can also be performed in the frequency and/or time domains for OFDM and MC-CDMA systems that provide full frequency diversity owing to the orthogonality between subcarriers of the OFDM modulation. OSTBC will be performed for multicarrier systems as described in [14] for different OSTBC codes.

\subsection{Linear Precoding with OFDM}

In order to apply linear precoding in the frequency domain, one may use a linear precoder of size $L \leq N_{c}$, where $\mathrm{L}$ always the size of the precoding matrix and $N_{c}$ is the 
number of subcarriers. This corresponds to an OFDM linear precoded scheme in the frequency domain. Again, in order to apply linear precoding in the time and frequency domains, one may use a linear precoder of size $L \geq N_{c}$. This corresponds to an OFDM linear precoded scheme in the time and frequency domains.

\subsection{Linear Precoding with $M C$-CDMA}

MC-CDMA combines OFDM modulation and CDMA access technique taking benefits from both the high spectral efficiency and the robustness against multipath channels of OFDM and access flexibility of CDMA [12][13]. In order to linearly precode a MC-CDMA scheme, we simply allocate codes for a specific user of length $L \geq N_{c}$. This means that allocation of spreading codes is carried out in the time and frequency domain. An interesting analogy can be made when MC-CDMA is applied with a linear precoding. In this case, MC-CDMA is equivalent to an OFDM scheme where our linear precoder is applied in both time and frequency domains. This can be adapted either to MIMO transmissions. We propose linear precoded OFDM scheme with OSTBC or linear precoded MC-CDMA scheme with OSTBC where temporal, spatial and frequency diversities are exploited.

\section{RESULTS AND CONCLUSION}

We carried out simulations in order to check the behaviour of the proposed system regarding the efficient exploitation of the diversities with multi-carrier systems, and to compare the performance with Hadamard-based and Fourier based precoders.

Figure 3 shows the performance of OSTBC with linear precoding for $L=32$ and spectral efficiency $\eta$ of $1 \mathrm{bps} / \mathrm{Hz}$ for a flat Rayleigh channel. To obtain $\eta=1$, a BPSK is applied to the Alamouti code, whereas QPSK is applied to Tarokh codes. For this spectral efficiency, the Alamouti performs worse than Tarokh codes, but this is not true for higher spectral efficiencies [3][14]. The results confirm that the performance improves with linear precoding for all tested OSTBC code providing a $2 \mathrm{~dB}$ gain at $\mathrm{BER}=10^{-3}$ with $\mathrm{G}_{4}$ with a very simple linear receiver. These results have been obtained with a number of transmit antennas $N_{t}$ corresponding to their respective OSTBC. Since the channel coefficients are uncorrelated, we find the same results by applying the OSTBC using subgroups depending on $N t$. For instance, we have the same results with the Alamouti code using $N t=2,4,8,16$ or 32 transmit antennas for $L=32$ and $N_{t} / 2$ subgroups if $N_{t}>2$.

Figure 4 shows the performance of the OSTBC Alamouti code $\left(N_{t}=2\right.$ and $\left.N_{r}=1\right)$ with linear precoded OFDM for $L=4$ or $L=4096$ and with $N_{c}=64$ over uncorrelated Rayleigh channels $(\eta=1)$. We see that the rotated Hadamard and FFT precoded OFDM give the same results when $N$ is large $(N=4096)$ but this specific Hadamard linear precoder performs better than FFT one when $\mathrm{L}$ is small $(L=4)$. Moreover, these results are similar to the results of MC-CDMA with OSTBC over Rayleigh channels, adding the benefits of the spreading in time and frequency dimensions. 


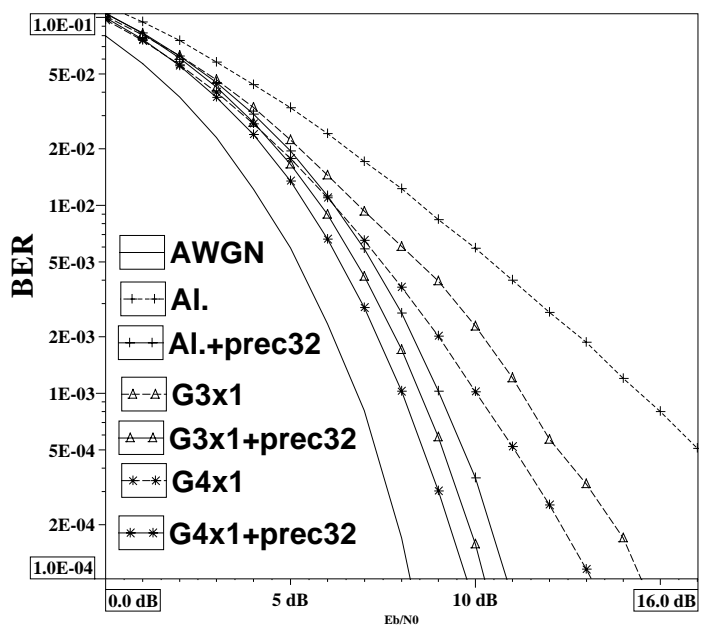

$\mathrm{L}=32$

$\eta=1 \mathrm{bps} / \mathrm{Hz}$

— with precoding

- - without precoding

Al: G2Alamouti code,

$\mathrm{R}=1$, for $N_{t}=2$

G3: Tarokh code

for $N_{t}=3$

G4: Tarokh code for $N_{t}=4$

Fig.3 Performance of STBC with linear precoding and different OSTBC

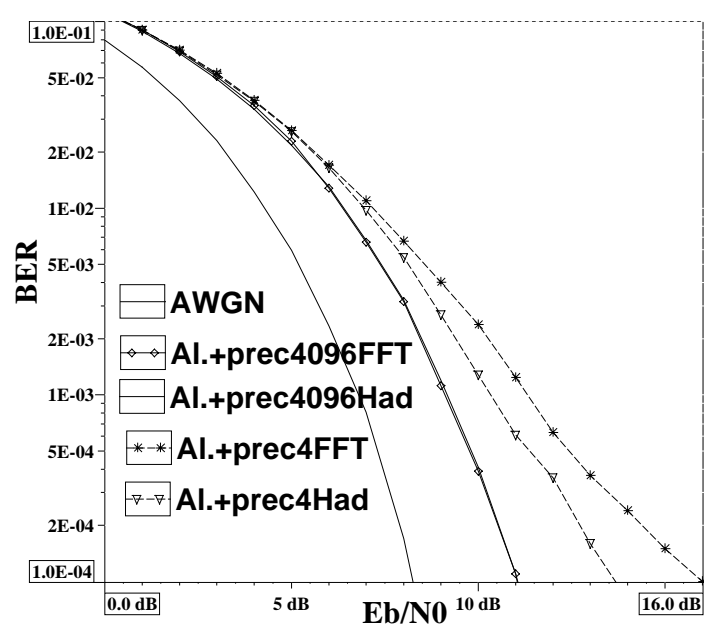

$$
\begin{aligned}
& --\mathrm{L}=4 \text { or } \\
& -\mathrm{L}=4096
\end{aligned}
$$

$\eta=1 \mathrm{bps} / \mathrm{Hz}$

Al: Alamouti code

Precoding matrix construction:

FFT: Fourier Transform Had: Hadamard matrix

Fig. 4. Performance of STBC with different linear precoding: Hadamard or Fourier

In this paper, we propose to linearly precode and decode OSTBC systems using a particular unitary matrix based on Hadamard or FFT construction. Our scheme has a low complexity, which only grows linearly with the size of the unitary matrix and not exponentially when more complex detectors are used. Simulation results with the specific linear precoders using OSTBC are given for flat independent Rayleigh 
fadings or OFDM systems. These precoders can be applied to various MIMO transmissions in order to exploit spatial, temporal and frequency diversities. We saw thanks to simulation results that the precoding method is very efficient for multicarrier modulations. We gave an interesting analogy between linear precoded OSTBC for flat independent Rayleigh fadings, linear precoded OFDM with OSTBC and linear precoded MC-CDMA with OSTBC. One can apply this linear precoding with any OSTBC, keeping the linearity of the transmission chain even at the receiver part. It is also possible to apply this linear precoding with QOSTBC, but at the expense of more complex receiver. Moreover, the proposed scheme suits to several multi-antenna configurations and thus can be adapted to channel characteristics.

\section{REFERENCES}

[1] Foschini G.J., Layered space-time architecture for wireless communication in a fading environment when using multi-element antennas, Bell Labs Tech. Journal, Vol. 1, No 2, pp. 41-59, 1996.

[2] Alamouti S.M, A simple Transmit Diversity Technique for Wireless Communications, IEEE Journal on Selected Areas in Communications, Vol. 16, No. 8, October 1998, pp. 1451-1458, 1998.

[3] Tarokh V., Jafarkhani H., and Calderbank A. R., Space-Time Block Codes from Orthogonal Designs, IEEE Transactions on Information Theory, Vol. 45, No. 5, pp. 1459-1467, July 1999.

[4] Jafarkhani H., A quasi-orthogonal space-time block code, IEEE Trans. Comm, 49, (1), pp. 1-4, 2001.

[5] Tirkkonen O., Boariu A., Hottinen A., Minimal orthogonality space-time block code for 3+ Tx antennas, Proc. IEEE Int. Symp. Spr. Spectr. Techn. Appl. (ISSSTA), New Jersey, USA, September 2000.

[6] Su W., Xia X., Two Generalized Complex Orthogonal Space-Time Block Codes of Rates 7/11 and 3/5 for 5 and 6 Transmit Antennas, to appear in IEEE Trans. on Inf. Theory, Jan. 2003

[7] Boutros J., Viterbo E., Signal Space Diversity: A Power and Bandwidth Efficient Diversity Technique for the Rayleigh Fading Channel, IEEE Trans. on Information Theory, Vol. 44, No.4, pp. 1453-1467, Jul. 1998.

[8] Damen M.O., K. Abed-Meraim, Belfiore J.C., Diagonal Algebraic space-time block codes, IEEE Trans. Inf. Theory, 48, (3), pp.628-636, 2002.

[9] Xin Y., Wang Z., and Giannakis G. B., Space-Time Diversity Systems Based on Unitary Constellation-Rotating Precoders, IEEE Conference on Acoustics, Speech, Systems and Signal Processing, Lake Louise, Alberta, Canada, pp. 396-401, Oct. 2000.

[10] Da Silva M. M., Correia A., Space-Time Block Coding for 4 antennas with Coding rate 1, IEEE $7^{\text {th }}$ Symp. On Spread Spectrum Technologies and Applications, Prague, Czech Republic, Sept. 2002.

[11] Le Nir V., Hélard M., Reduced-Complexity Space-Time Block Coding and Decoding schemes with block linear precoding, IEE Electronic letters, Vol. 39 No.14, 10 $0^{\text {th }}$ July 2003.

[12] Yee N., Linnartz J.P, Fettweis G., Multicarrier CDMA in Indoor Wireless Radio Networks, IEEE PIMRC'93, pp. 109-113, Yokohama, Japan, 1993.

[13] Hélard M. , Le Gouable R., Hélard J.F, Baudais J.Y., Multicarrier techniques for future wideband wireless network, Annales des Télécom Numéro spécial UMTS, Vol. 56, N5-6, pp. 260-274, 2000.

[14] Le Nir V., Hélard M., Le Gouable R., Space-Time Block Coding Applied to Turbo Coded Multicarrier CDMA, Vehicular Technology Conference, Jeju, South Korea, 22-25 April 2003.

[15] IST MATRICE project, web site http://www.ist-matrice.org.

\section{AFFILIATIONS}

The authors work at France Télécom R\&D in DMR/DDH lab. For more detailed informations, you can contact them at the following email addresses:

\{vincent.lenir;maryline.helard;rodolphe.legouable\}@ francetelecom.com

Part of this work has been carried out in the IST-MATRICE project [15]. 